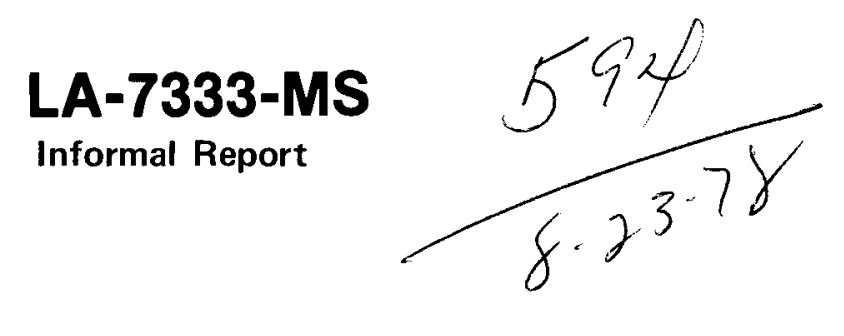

th. 390 LA-7333-MS 594.

$8 \cdot 23$

算罢

\title{
A Magnetic Induction Technique for Mapping Vertical Conductive Fractures: \\ Theory of Operation
}

$\frac{\pi}{\frac{\sigma}{6}}$

$\mathcal{O}$

¿

$\frac{7}{\omega}$

交 


\section{DISCLAIMER}

This report was prepared as an account of work sponsored by an agency of the United States Government. Neither the United States Government nor any agency Thereof, nor any of their employees, makes any warranty, express or implied, or assumes any legal liability or responsibility for the accuracy, completeness, or usefulness of any information, apparatus, product, or process disclosed, or represents that its use would not infringe privately owned rights. Reference herein to any specific commercial product, process, or service by trade name, trademark, manufacturer, or otherwise does not necessarily constitute or imply its endorsement, recommendation, or favoring by the United States Government or any agency thereof. The views and opinions of authors expressed herein do not necessarily state or reflect those of the United States Government or any agency thereof. 


\section{DISCLAIMER}

Portions of this document may be illegible in electronic image products. Images are produced from the best available original document. 
This report was prepared as an account of work sponsored by the United States Government. Neither the United States nor the United States Department of Energy, nor any of their employees, nor any of their contractors, subcontractors, or their employees, makes any warrancy, express or implied, or assumes any legal liabilly or responsibility for the accuracy. completeness, or usefulness of any information, apparatus. product, or process disclosed. or represents that its use would not infringe privately owned rights.

UNITED STATES DEPARTMENT OF ENERGY CONTRACT W-7403-ENG. 36 
LA-7333-MS

Informal Report

UC-66b

Issued: July 1978

齐 1

1.

\title{
A Magnetic Induction Technique for Mapping Vertical Conductive Fractures: Theory of Operation
}

\author{
J. A. Landt
}

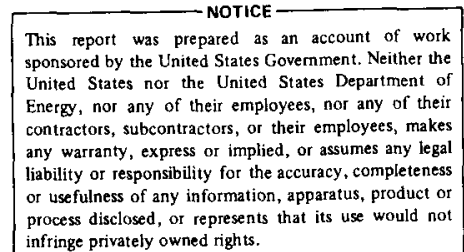




\section{A MAGNETIC INDUCTION TECHNIQUE FOR MAPPING \\ VERTICAL CONDUCTIVE FRACTURES: \\ THEORY OF OPERATION}

\section{J. A. Landt}

\section{ABSTRACT}

Utilization of a hot dry rock geothermat resource requires circulation of a fluid (water) through fractures in the rock. The Los Alamos Scientific Laboratory is presently investigating the feasibility and economics of tapping this energy resource. Presently, the fractures in the rock are created by conventional hydraulic fracturing techniques. Accurate determination of the geometry of the fracture system is required so that boreholes may be drilled to complete the circulation system. This report presents the theory of a technique designed to map vertical conductive fractures located in resistive rock. The technique is based on magnetic induction. Fracture thickness and strike can be determined from measurements made in a single borehole.

\section{INTRODUCTION}

As part of the country's search for alternative energy sources, the Los Alamos Scientific Laboratory (LASL) is investigating techniques for extracting energy from hot dry rock. ${ }^{1}$ The experiment currently under way is schematically shown in Fig. 1. The proposed sequence consists of: (1) drilling a hole into a hot, solid rock formation; (2) hydraulically fracturing the rock; (3) determining the fracture orientation; (4) intersecting the fracture with a second drill hole; and (5) circulating a working fluid (water) to extract thermal energy. This sequence is being attempted in a geothermal region of the Jemez Mountains in northern New Mexico near Los Alamos. The results of these efforts have been reported elsewhere, and the interested reader is referred to the literature.

The present experiment has confirmed the prediction that a single vertical fracture will be produced at depths $(\sim 3000 \mathrm{~m})$ where the temperature is high enough $\left(\sim 200-250^{\circ} \mathrm{C}\right)$ to make the proposed scheme viable. The present experiment has pointed to many deficiencies in measurement techniques at these elevated temperatures, and also to a general lack of fracture mapping techniques that could per- mit directional drilling of the second hole with sufficient accuracy to intercept the fracture.

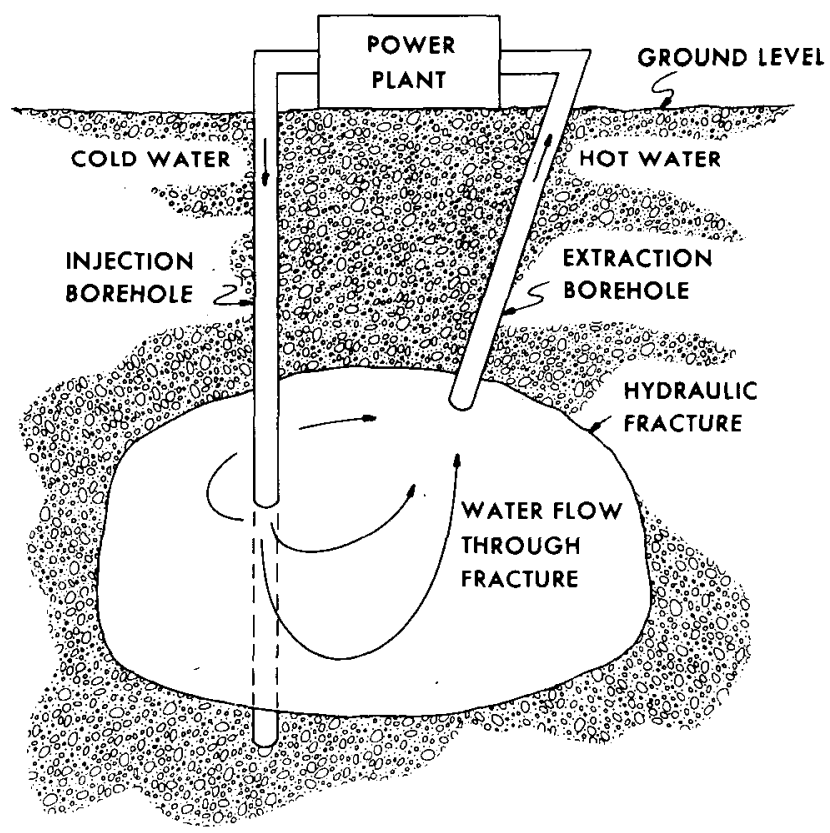

Fig. 1. The extraction of energy from hot dry rock using hydraulic fracturing. 
This report addresses the design of an instrument capable of mapping vertical conductive fractures in a resistive bedrock. The fracture is mapped using magnetically induced eddy currents and the instrument is quite similar to the induction sondes employed by the $0 i 1$ industry. ${ }^{2,3}$ Sufficient differences exist between these existing devices and the proposed instrument, however, to warrant a detailed look at the theoretical response of the proposed device. Programmatic requirements have not permitted a study with the breadth and depth needed to utilize the full potential of the instrument. Consequently, what follows here is an investigation with sufficient detail to allow design of a prototype instrument. Much further work will be required to optimize the design and study and influences of all of the important parameters.

\section{BACKGROUND}

The experiment shown in Fig. 1 has been attempted at Fenton Hill near Los Alamos. While some difficulty has been encountered in establishing a low-impedance connection between the two boreholes, experiments on the measurements of this existing system have provided much detail. The fractures appear to be roughly circular vertical disks of several hundred meters in diameter and several militmeters wide. The fractures are at a depth of about $3000 \mathrm{~m}$. Future requirements indicate that the proposed instrument will be required to work in an environment of hot water of $275^{\circ} \mathrm{C}$ at a pressure of $10000 \mathrm{psi}\left(7 \times 10^{7} \mathrm{~Pa}\right)$. The circulation fluid is obtained locally from a fresh water stream. This water has a resistivity of $\sim 10 \Omega-\mathrm{m}$ at $250^{\circ} \mathrm{C}$. If the fracture width is $3 \mathrm{~mm}$, the fracture corresponds to an equivalent sheet resistivity of $3000 \Omega$ /square. The resistivity of the surrounding rock is greater than $3000 \Omega-m$.

The fracture zone has been measured with a wide variety of techniques. Some of these are listed here:

1. Control of fracture zone. High-pressure packers are used to select the region where the fracture will begin.

2. Spinners. This conventional device determines where fluid is entering or leaving the well bore. 4
3. Tracers. Various tracers have been used to study fluid flow and confirm connection.

4. Downhole electrical surveys. Comparison of measurements taken before and after fracturing point to anomalous regions affected by the fracturing process.

5. Calipers. Potentially, a caliper device would permit one to "feel" the fracture. A hightemperature caliper with sufficient resolution is not yet available.

6. Impression packers. These devices have been used, but are difficult, to interpret and permit investigation of only small sections of borehole.

7. Borehole televiewer. This device works quite well. It is basically an ultrasonic ranging device. A high-temperature version has detected fractures at Fenton $\mathrm{Hill}$.

8. Two-hole seismic. This technique has been used extensively at Fenton Hill. 5,6 The present picture of the fracture was made using this technique. Basically, a geophone array is lowered in a borehole and microearthquakes are detected as the fracture is inflated and deflated. The biggest drawback is the need for a second hole, although in principle, the instrument can be used in a single borehole to map the fracture far from the borehole.

9. Current injection. Fracture zones have been mapped by injecting current downhole and measuring ground potentials around the well bore. ${ }^{7}$ This method appears to be limited to shallow fracture zones.

10. Single-hole acoustic. No equipment exists yet, but the device has been patented. This scheme depends on placing acoustic transmitters and receivers around the surface of the borehole. $S$ and $P$ waves propagate differently across a fracture. This device measures this and thus locates the fracture intersection at the borehole.

11. Television. It is possible to lower a television camera downhole and look at the borehole surface. This works only with a clear drilling fluid and, consequently, has not been used extensively by the oil industry.

12. Magnetic material injection. This scheme relies on perturbing the earth's magnetic field. ${ }^{8}$ No equipment has been built yet. The injection of 
foreign material into the fracture may be objectionable.

The $0 i 1$ industry uses several other techniques to attempt to measure fractured systems, but generally, these do not lend themselves to the singlefracture system that is to be measured in hot dry rock geothermal systems. Schemes 1 through 4 do not map fracture direction, and the remainder that are applicable to deep fractures (except for the twohole scheme) determine only the fracture geometry at the borehole surface. This may not reflect the true fracture orientation.

The technique discussed in this report permits fracture mapping away from the borehole, using a single borehole, and at any depth. The technique was originally proposed several years ago at LASL by P. R. Kintzinger, F. G. West, and W. Johnson, but it was not actively pursued until recently. The analytic details of this technique are the topic of this report. In principle, this device is similar to the induction $\log$ used by the $0 i 1$ industry. ${ }^{2,3}$ conceptually, magnetic fields are set up in a conducting medium that drive eddy currents. These eddy currents, in turn, drive magnetic fields that are sensed by a receiver. This permits mapping into the formation. Several difficulties arise for the intended application, however, and these are dealt with later. Theoretical problems arise in handling the borehole signal. Practical difficulties include detection of a signal on the order of several tens of nanovolts, tradeoffs between instrument size and the depth of investigation, and details in construction to eliminate spurious signals. Unlike the conventional induction devices, the present instrument does not have to be accurately calibrated in an absolute sense.

\section{BASIC EQUATIONS FOR THE RESPONSE OF MAGNETIC INDUCTION FRACTURE MAPPER}

The response of the instrument can be found using the geometry shown in Fig. 2 . The borehole axis is along the $Y$-axis and the borehole has been omitted at present for clarity and simplicity. A coil-carrying current, $I_{t}$, is shown at the origin lying in the $X Y \mathrm{plane}$ with its axis along the Zaxis. Conventional spherical coordinates are used. $A$ vertical fracture is also depicted, and this fracture is rotated an angle $\beta$ from the $X Y$ plane. The

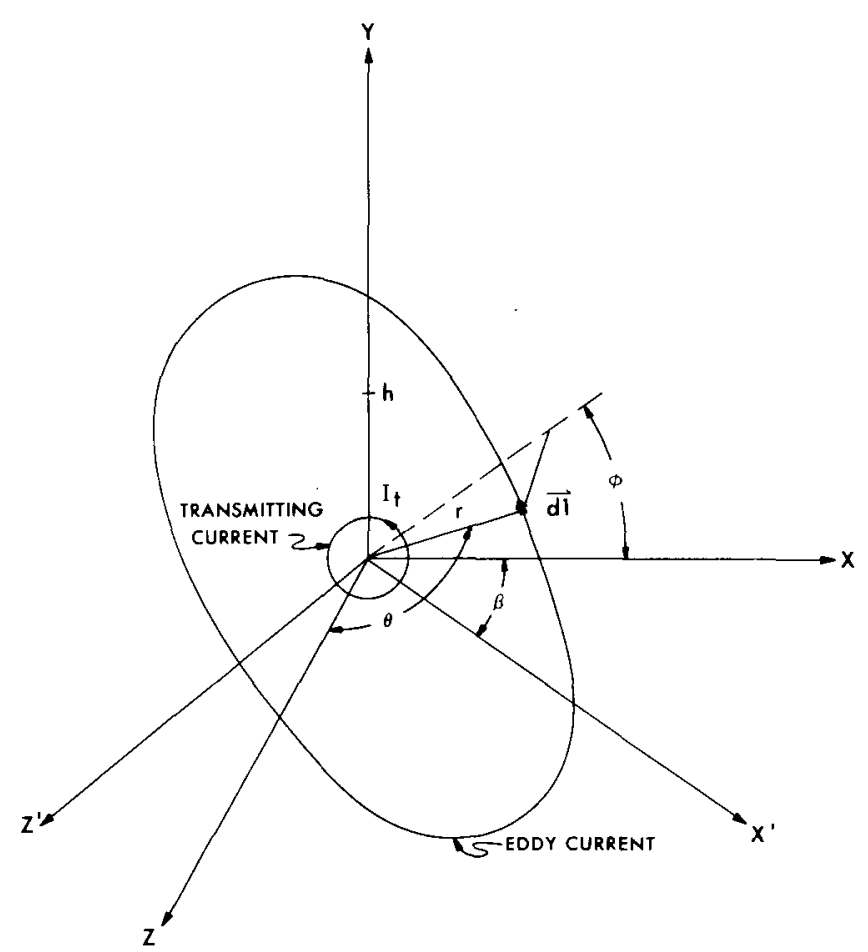

Fig. 2. Geometry of a current loop and an eddy current loop.

fracture lies in the X'Y plane. A single circular eddy current loop is also shown. The point $h$ is a point on the $Y$-axis (in the borehole) where a receiving coil will be placed. The total magnetic field is of interest here.

The response of the instrument can be found by using the fields of a magnetic dipole in conjunction with Ohm's law and Ampere's law.

- The electric and magnetic fields surrounding a magnetic dipole (or small coil carrying an electric current) are ${ }^{9}$ :

$$
\vec{E}(\vec{r})=\eta \frac{N S I}{4 \pi} e^{-j k r}\left(\frac{k^{2}}{r}-\frac{j k}{r}\right) \sin \theta \hat{a}_{\phi}
$$

$\vec{H}(\vec{r})=\frac{N S I}{4 \pi} e^{-j k r}\left[2\left(\frac{j k}{r^{2}}+\frac{1}{r^{3}}\right) \cos \Theta \hat{a}_{r}\right.$

$$
\left.+\left(-\frac{k^{2}}{r}+\frac{j k}{r^{2}}+\frac{1}{r^{3}}\right) \sin \theta\right] \hat{a}_{\theta},
$$


where $n$ is the impedance of the medium, $N$ the number of turns in the coil, $S$ the area of the coil, I the current in the coil, $k$ the wave number, and $r$, $\theta$ and $\phi$ are shown in Fig. 2. Conventional spherical coordinate unit vectors $\hat{a}_{r}, \hat{a}_{\phi}$, and $\hat{a}_{\Theta}$ are used. For this report, quasistatic solutions will be used (Ampere's law assumes this also). Equation (1) can be simplified to the quasistatic case by keeping only first-order terms as $\mathrm{kr} \rightarrow 0$. If this is done formally by expanding $e^{j k r}$, the quasistatic electric field is found to be:

$\vec{E}(\vec{r})=-j \frac{k n N S I \sin \theta}{4 \pi r^{2}} \hat{a}_{\phi}$.

Using the identities $k=\omega / c=\omega \sqrt{\mu \varepsilon}$ and $\eta=\sqrt{\mu / \varepsilon}$, where $\varepsilon$ is the permittivity and $\mu$ the permeability of the medium, $\vec{E}$ can be written:

$\vec{E}(\vec{r})=-j \frac{\omega \mu N S I \sin \theta}{4 \pi r^{2}} \hat{a}_{\phi}$.

Here, $\omega$ is the angular frequency in radians/second and $j a \sqrt{-1}$.

If the medium is not too conductive, Eq. (3) can be used to describe the electric field in that medium. The eddy currents can be found using ohm's law:

$$
\vec{J}=\sigma \vec{E}
$$

The currents display the same symmetries as the electric field. This knowledge of the current distribution permits the use of Ampere's law to calculate the magnetic fields due to the eddy currents (see Fig. 3.):

$\vec{H}(\vec{r})=\frac{1}{4 \pi} \delta_{V} \frac{I\left(r^{\prime}\right) \vec{d}^{\prime} \times\left(\vec{r}-\vec{r}^{\prime}\right)}{\left|\vec{r}-\vec{r}^{\prime}\right|^{3}}$

The observation point is at $\vec{r}$, and the source at $\vec{r}^{\prime}$. Here, the small arrows, $\rightarrow$, denote vector quantities.

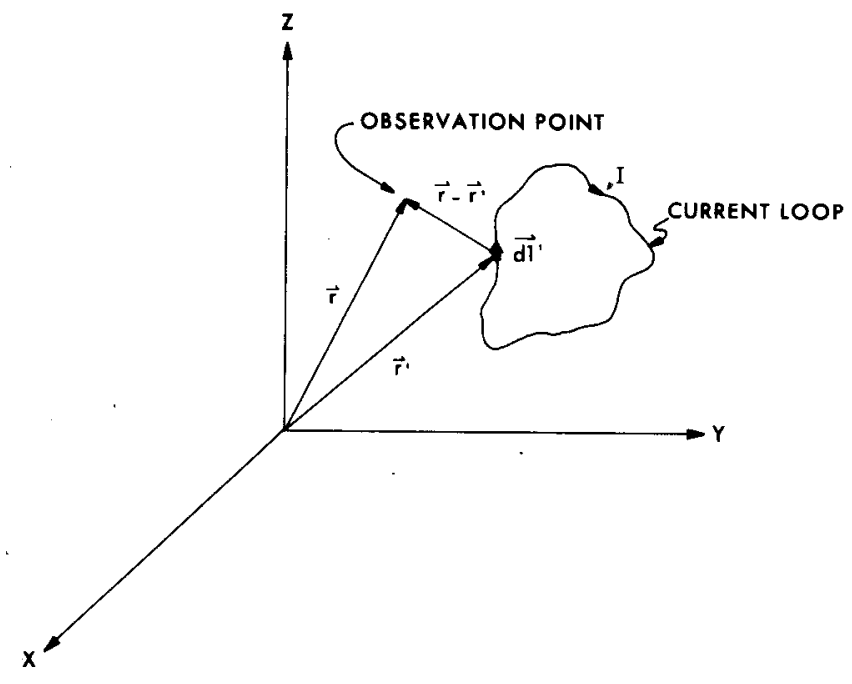

Fig. 3. Conventions used for the application of Ampere's law.

A qualitative idea of the response of the instrument can be found by considering the single eddy current loop shown in Fig. 2. (A complete response is simply the summation of all such eddy current loops.) Analysis will be eased by using superposition. The angle between the plane of the eddy current loop and the plane of the coil is $\beta$. The single coil may be considered the sum of two perpendicular coils. Choose these equivalent coils so that one of them lies in the X'Y plane and the other in the $X Z^{\prime} p$ lane. The original magnetic moment is:

$\vec{M}=N S I \hat{a}_{z}$.

Break $\vec{M}$ up into the required components:

$\vec{M}=\operatorname{NSI}\left(\cos \beta \hat{a}_{z^{\prime}}+\sin \beta \hat{a}_{x^{\prime}}\right)$.

In Eq. (3), $\theta$ is measured from the axis of the coil (the $z$-axis in this case). Consequently, the electric field around the eddy current loop is:

$\vec{E}(\vec{r})=-j \frac{\omega \mu N S I \cos B}{4 \pi r^{2}} \AA_{\phi^{\prime}}$. 
If the total resistance of the loop is $R$, then by Ohm's law, the current in the loop is

$I=V / R$,

where

$V=\int \vec{E} \cdot \overrightarrow{d l}$

Combining,

$I=-j \frac{\omega \mu N S I \cos B}{2 R}$,

or

$I=I_{0} \cos \beta$.

Because of symmetry and using Ampere's law, the magnetic field along the $Y$-axis will be in the $Z^{\prime}$ direction. Consider qualitatively what this field will be as a function of $\beta$. The angle $B$ determines the magnitude of the eddy currents. Subsequent application of Ampere's law to find the magnetic field at a point on the $Y$-axis a length $h$ from the origin involves the current in the loop, the size of the loop $r$ and the dimension $h$. Combining the $r$ and $h$. variations into a yet undetermined function $F(r, h)$, the magnetic field at point $h$ is

$\vec{H}_{R}=I_{0} F(r, h) \cos \beta \hat{a}_{z^{\prime}}$.

If this field is measured by a small coil at $h$ and parallel to the first coil, the voltage induced will be:

$V=-n \frac{d \Phi}{d t}$

$$
\begin{aligned}
& =-j \omega n s I_{0} F(r, h) \cos ^{2} \beta \\
& =V_{0} \cos ^{2} \beta,
\end{aligned}
$$

where $n$ and $s$ are the number of turns and area of the coil at $h$, respectively.

The equations of this section will be used later to calculate the response of proposed instrument configurations. At that time, the details omitted here [specifically in Eq. (11)] will be provided.

IV. QUALITATIVE RESPONSE

The heart of this instrument lies in Eq. (12c). There it is seen that a two-coil instrument will have a response as is shown in Fig. 4 as the two coils are rotated together. The $\cos ^{2} \beta$ function is a maximum when the coils are lined up with the fracture and is a minimum when they are perpendicular to the fracture. Conceptually, the simplest fracture mapper would consist of two parallel coils held one above the other in the borehole and with their axes in a horizontal direction. As the coils are rotated together, a null would indicate that the coils are perpendicular to the fracture and, conversely, a maximum indicates that the coils are in the plane of the fracture.

A. Mültiple Fractures

Equation (12c) can be used to study the instrument response to multiple fractures. Consider a series of $N$ fractures that individually provide received signal strengths of $V_{i}$. If these fractures are embedded in a nonconductive medium, and if the eddy currents in each fracture are small (implying low or moderate fracture conductivity) so that the eddy current distribution in each fracture is

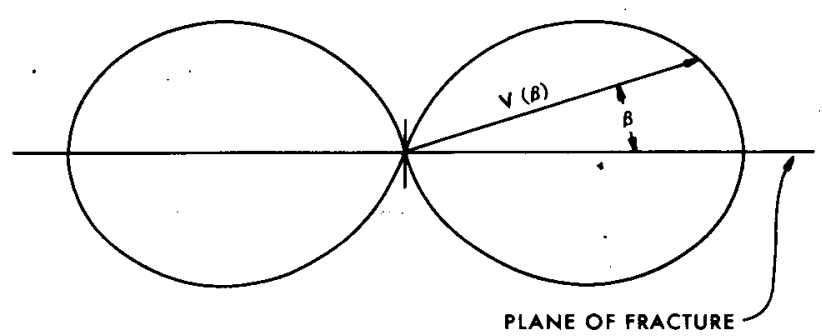

Fig. 4. Idealized detector response as a function of the angle ( $B$ ) between the plane of the fracture and the plane of the coils. 
unaltered. then the total signal is the sum of signals due to each fracture considered separately:

$$
\begin{aligned}
V(\beta)= & V_{i} \cos ^{2}\left(\beta-\alpha_{1}\right) \\
& +V_{2} \cos ^{2}\left(\beta-\alpha_{2}\right)+\cdots+v_{N} \cos ^{2}\left(\beta-\alpha_{N}\right),
\end{aligned}
$$

where $\alpha_{i}$ is the angle of the $i^{\text {th }}$ fracture. Through algebraic manipulation, it can be shown that

$V(\beta)=C^{\prime}+V^{\prime} \cos ^{2}\left(\beta-\alpha^{\prime}\right)$,

where

$$
C^{\prime}=-\frac{V^{\prime}}{2}+\sum_{i=1}^{N} \frac{V_{i}}{2}
$$

$V^{\prime}=\sqrt{\left(\sum_{i=1}^{N} v_{i} \cos 2 \alpha_{i}\right)^{2}+\left(\sum_{i=1}^{N} v_{i} \sin 2 \alpha_{i}\right)^{2}}$,

and

$$
\alpha^{\prime}=\frac{1}{2} \tan ^{-1}\left(\frac{\sum_{i=1}^{N} v_{i} \sin 2 \alpha_{i}}{\sum_{i=1}^{N} v_{i} \cos 2 \alpha_{i}}\right) \text {. }
$$

A basic theoretical limitation of this instrument is indicated by Eq. (13b). The fine structure of multiple-fractured systems cannot be resolved. For example, for two fractures of equal individual response, the instrument will have a maximum response when the coils are bisecting the angle between the two fractures. If two fractures are present, it can be shown that the "pointing error" for determining the direction of the larger of the two is: $\alpha_{\text {error }}=\frac{1}{2} \tan ^{-1}\left(\frac{v_{S}}{\sqrt{v_{l}^{2}+v_{S}^{2}}}\right)$,

where $V_{\ell}$ is the signal provided by the large fracture alone, and $V_{S}$ provided by the small fracture. For example, a 10-to-1 ratio in fracture size would result in a maximum pointing error of $2.9^{\circ}$. If the near borehole region contains many small fractures, these will tend to give a uniform "background" signal and permit mapping of the large fracture.

\section{B. Electronic Coil Rotation}

Instead of mechanically rotating the coil set, it is possible to synthesize rotation electronicaliy. Electronic rotation can be accomplished at least two ways. Either of these schemes relies on two sets of coils, one set mounted perpendicular to the other set, as shown in Fig. 5 . Rotation can be accomplished by controlling the magnitude of the current in each transmitter coil and then appropriately combining the signal measured by the receiving coils. If rotation to an angle $B$ is desired, then the current in coil \#1 is

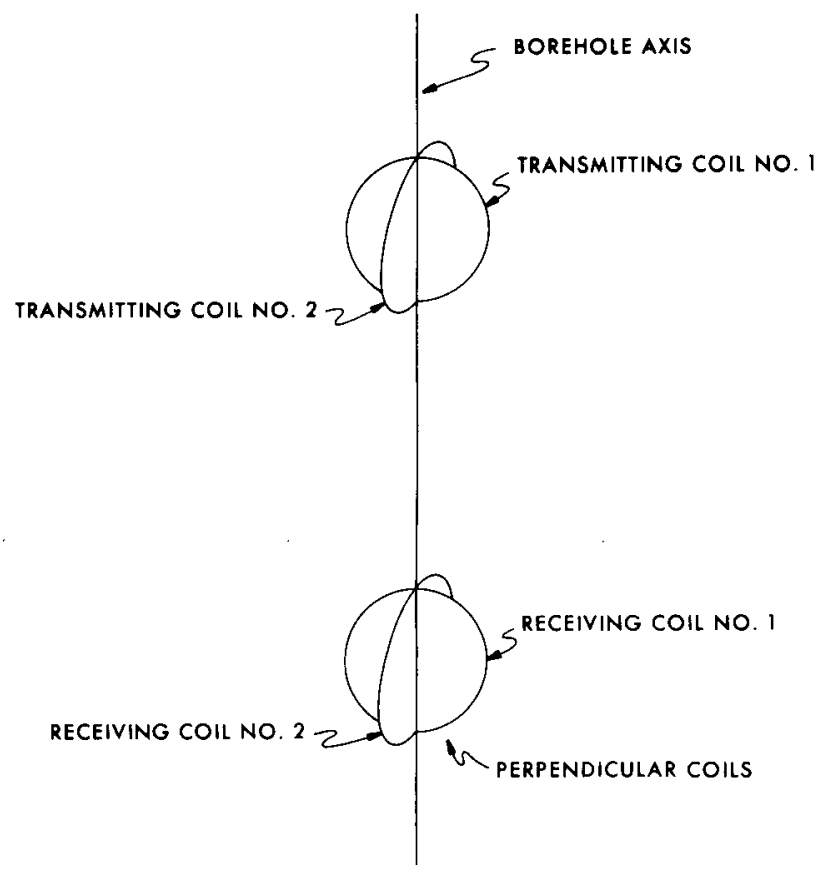

Fig. 5. Geometry of a "two-coil set" instrument for electronic rotation. 
$I_{1}=I_{0} \cos \beta$

and the current in coil \#2 is

$I_{2}=-I_{0} \sin \beta \quad$.

These combined coils produce a magnetic field that is the same as a coil at angle $B$ carrying a current $I_{0}$. The signals received in the two coils are combined into a single signal following the formula:

$v_{r}=v_{1} \cos \beta-v_{2} \sin \beta$.

This combination results in the same received signal as a single receiving coil oriented at an angle $\beta$.

While the control outlined above is simple in concept, it requires a great deal of accuracy and stability. A scheme that is easier to implement electronically involves a two-part experiment. First, a current is driven in transmitter coil \#1 and signals are measured in both of the receiving coils. Define "impedances" according to the formulas:

$Z_{11}=\frac{v_{r l}}{I_{t]}}$

$Z_{21}=\frac{V_{r 2}}{I_{t 1}}$.

Then excite a current in transmitter coil \#2 and form the impedances:

$z_{12}=\frac{v_{r l}}{I_{t 2}}$

$$
Z_{22}=\frac{V_{r 2}}{I_{t 2}}
$$

These impedances may be calculated at the surface once the measurements are made, thus eliminating an "electronic divider." Since the system is linear, $Z_{12}=Z_{21}$ and this can be used for diagnosis of electronics performance. The impedances of Eqs. (16a) - (16d) contain the information required to synthesize a mechanical rotation. This is done by combining them according to the formula to find the voltage at an angle $\beta$ :

$$
\begin{aligned}
V_{r}(\beta)= & {\left[\left(Z_{11} \cos \beta-Z_{12} \sin \beta\right) \cos \beta\right.} \\
& \left.-\left(Z_{21} \cos \beta-Z_{22} \sin \beta\right) \sin \beta\right] I_{0},
\end{aligned}
$$

where $B$ is measured from the plane of coils labeled \#1. The proof that this provides the desired result is straightforward. For example, consider a single conductive fracture in a background conductive medium. For each transmitting coil, the fields can be broken into components parallel to and perpendicular to the fracture. Considering these separately and using superposition, it can be shown that if the coil sets are identical in construction and location except for a $90^{\circ}$ rotation, the impedances are:

$$
\begin{aligned}
& z_{11}=z_{R}+z_{F} \cos ^{2} \alpha \\
& z_{12}=-z_{F} \sin \alpha \cos \alpha \\
& z_{21}=-z_{F} \cos \alpha \sin \alpha
\end{aligned}
$$

$z_{22}=z_{R}+z_{F} \sin ^{2} \alpha$

where

$$
Z_{F}=\frac{V_{F}}{I_{0}},
$$

and

$$
Z_{R}=\frac{V_{R}}{I_{0}},
$$


where $a$ is the angle between the fracture and the coils labeled 1, $I_{0}$ is defined in Eq. (11) and $V_{F}$ in Eq. (12c). $V_{R}$ is the signal due to a homogenous background and is described later. Substituting these values in Eq. (17), and simplifying:

$v_{r}(\theta)=\left[Z_{B}+Z_{F} \cos ^{2}(\beta-\alpha)\right] I_{0}$,

$(18 \mathrm{~g})$

which is the same form as Eq. (13b). Extension to multiple fractures is straightforward. If only the direction of the fracture is desired, that can be found from:

$\alpha=\frac{1}{2} \tan ^{-1}\left(-\frac{z_{12}+z_{21}}{z_{11}-z_{22}}\right)$.

This equation is easily verified using the $Z^{\prime} s$ of Eq. (18). Notice that the sign of $z_{12}$ 'and $z_{21}$ contain the information about the quadrant of $\alpha$. The electronics must be designed with this in mind.

These equations also point to the need for extreme care in construction to eliminate spurious signals. Crosstalk between a transmitter coil and the receiver coil, which is perpendicular, is especially critical. This crosstalk will limit the sensitivity of the instrument.

The discussion in this section forms the basis for a detailed investigation of the response of the instrument. Items to be considered later are the specific responses for a variety of configurations and rock and fracture properties. Effects such as fracture offset and tip will be addressed in future work as well as consideration of borehole signals.

\section{TWO-COIL INSTRUMENT RESPONSE}

A. Infinite Medium Response

Superposition can be used to calculate the response of a multiple-coil instrument from the twocoil response. First, the response of a two-coil instrument immersed in a homogeneous medium will be addressed. 10,11 This is to provide insight as well as calculate the response of the fracture mapper to the relatively nonconductive bedrock.

The voltage induced in a single turn-receiving coil can be calculated using Faraday's law:
$V=-\frac{\partial}{\partial t} \int_{S} \vec{B} \cdot \overrightarrow{d S}$

If it is assumed that the receiving loop is small so that $\vec{B}$ is uniform across the surface of the loop, Eq. (19) reduces to

$V=-j \omega \mu S_{R} H_{n}$,

where $H_{n}$ is the magnetic field component normal to the surface of the loop and $S_{R}$ is the area of the loop. For a multiturn coil of $N_{R}$ turns, the tota] induced voltage is

$V=-j \omega \mu N_{R} S_{R} H_{n}$

If the receiving coil is at $y=R$ on the $Y$-axis with the coil axis pointing in the $Z$-direction and using $\mathrm{H}$ from $\mathrm{Eq}$. (1b), the voltage becomes

$V_{R}=j \frac{N_{T} S_{T} N_{R} S_{R} I_{T}(\omega) \mu}{4 \pi}$

$$
x e^{-j k R}\left[-\frac{k^{2}}{R}+j \frac{k}{R^{2}}+\frac{1}{R^{3}}\right] \text {. }
$$

The wave number, $k$, is

$k=\omega \sqrt{\mu \varepsilon} \sqrt{1-j \frac{\sigma}{\omega \varepsilon}}$.

In these equations,

$\mathrm{N}_{\mathrm{T}}=$ number of turns on the transmitting coil

$N_{R}=$ number of turns on the receiving coil

$S_{T}=$ area of the transmitting coil

$S_{R}=$ area of the receiving coil

$I_{T}=$ current in the transmitting coil

$\omega=$ angular frequency in $\mathrm{radians} / \mathrm{second}$ where frequency in $\mathrm{Hz}=f=\omega / 2 \pi$

$\mu=$ permeability of the medium, $\mu=4 \pi \times 10^{-7}$ $\mathrm{H} / \mathrm{m}$ for free space

$\varepsilon=$ permittivity of the medium, $\varepsilon=10^{-9} / 36 \pi$ $\mathrm{F} / \mathrm{m}$ for free space 
$\sigma=$ the conductivity of the medium in mhos $/ \mathrm{m}$

$R=$ the distance between the coils.

It should be stated again that Eqs. (1) and

(21) are valid only for small coils. That is, where $\mathrm{R}$ is much larger than the coil diameter.

Response of the two-coil instrument for various combinations of $\omega, \varepsilon$, and $\sigma$ has been given in graphical form by Fuller and wait. 11 For the present application, however, Eq. (21) will be reduced further with assumptions of the material to be mapped. Using the series $e^{x}=1+x / 1 !+x^{2} / 2 !+x^{3} / 3 !+\ldots$, Eq. (21c) can be reduced to

$$
\begin{aligned}
V_{R}= & j \frac{N_{T} S_{T} N_{R} S_{R} I-\omega \mu}{4 \pi R^{3}}\left[1-\frac{1}{2} R^{2} k^{2}\right. \\
& +R^{3}(j k)^{3}\left(-\frac{1}{3 !}+\frac{1}{2 !}-\frac{1}{1 !}\right) \\
& +R^{4}(j k)^{4}\left(\frac{1}{4 !}-\frac{1}{3 !}+\frac{1}{2 !}\right) \\
& \left.+R^{5}(j k)^{5}\left(-\frac{1}{5 !}+\frac{1}{4 !}-\frac{1}{3 !}\right)+\cdots\right],
\end{aligned}
$$

or, combining to

$$
\begin{aligned}
V_{R}= & \frac{N_{T} S_{T} N_{R} S_{R} T^{\omega \mu}}{4 \pi R^{3}}\left[1-\frac{1}{2} R^{2} k^{2} \mu \varepsilon\left(1-j \frac{\sigma}{\omega \varepsilon}\right)\right. \\
& \left.-\frac{2}{3} R^{3}(j k)^{3}+\cdots\right] .
\end{aligned}
$$

If a quasistatic condition is defined when $|R k|<<1$, then under quasistatic conditions,

$$
\begin{aligned}
V_{R}= & j \frac{N_{T} S_{T} N_{R} S_{R} I_{T} \omega \mu}{4 \pi R^{3}}\left[\left(1-\frac{R^{2} \omega^{2} \mu \varepsilon}{2}\right)\right. \\
& \left.+j R^{2} \frac{\omega \mu \sigma}{2}\right] .
\end{aligned}
$$

Equation (23c) forms the basis for commercial induction devices. Measurement of the received signal in phase with the transmitted current is directly proportional to the material conductivity.

Equation (23c) was derived here to be used to find the response of the background rock and to provide further insight of the physical behavior of the response. Notice that in an infinite homogeneous medium, the response is independent of the orientation of the coils as long as the coils are held fixed with respect to each other. While the method pursued in this section is fairly straightforward and provides the response in a homogeneous medium, the method is not capable of analyzing the response obtained from a thin fracture. The response of a thin fracture and also of a homogeneous medium can be found with the use of Eqs. (1a), (4), (5), and (12a) or (20).

This alternate approach was discussed briefly in the last section, where the dependence of coil orientation with respect to the fracture was studied. Here, this approach will be developed to obtain the instrument response in a homogeneous medium so that a check with Eq. (23c) can be made. Discussion of fracture response will be deferred until the next section.

The application of Ohm's law technique outlined earlier hinges on the ability to subdivide a region into elements, where the currents are, not altered in magnitude or direction from that given by $\vec{J}=\sigma \vec{E}$. It is also assumed that the field $\vec{E}$ is given and is not a function of the currents. Therefore, the following analys is is limited to regions of low or moderate conductivity. A complete boundary value solution is required for regions of high conductivity or unusual shape.

Consider the eddy current loop shown in Fig. 6. The axis of this loop is the Z-axis. (The transmitting coil is located at the origin. The axis of the transmitting coil is also along the Zaxis.) The distance between the transmitting coil and the plane of the eddy current loop is $Z$. The radius of the eddy current loop is $\rho$. An observer is at point 0 , a distance $R$ from the origin along the $Y$-axis. Equation (20) can be used to find the received signal if $\mathrm{H}_{Z}$ is found at point 0 From Eqs. (3) and (4), 


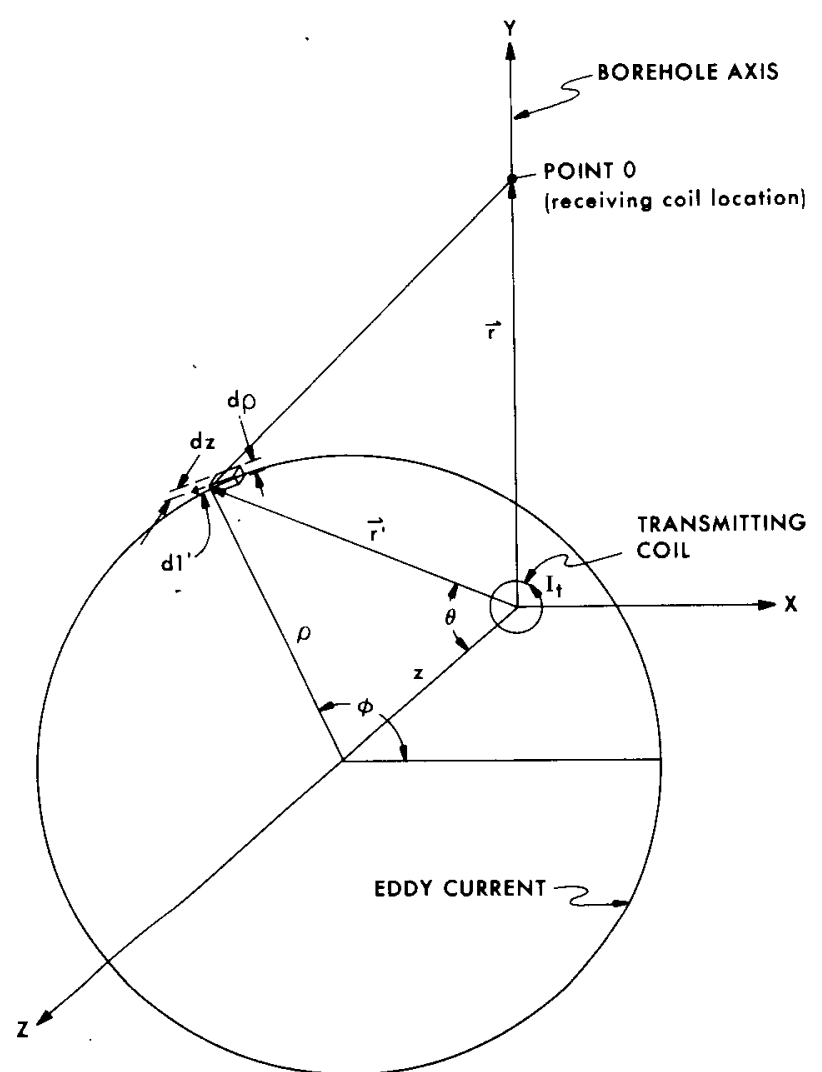

Fig. 6. Geometry of an eddy current loop offset from the borehole.

$\vec{j}=-j \frac{\omega \mu \sigma N_{T} S_{T} I_{T} \sin \theta}{4 \pi\left(r^{\prime}\right)^{2}} \hat{a}_{\phi}$,

where

$\sin \theta=\rho / r^{\prime}$

$\vec{r}^{\prime}=\rho \cos \phi \hat{a}_{x}+\rho \sin \phi \hat{a}_{y}+z \hat{a}_{z}$

$\vec{r}=R \hat{a}_{y}$

$\overrightarrow{d l^{\prime}}=\frac{\vec{j}}{|\vec{J}|}=\hat{a}_{\phi}=\rho d \phi\left(-\sin \phi \hat{a}_{x}+\cos \phi \hat{a}_{y}\right)$.

The total current in the loop is

$$
\begin{aligned}
& I=|\vec{j}| d S \\
& I=|\vec{J}| \mathrm{d} \rho \mathrm{d} z \\
& \text { Also, } \\
& \vec{d}^{\prime} \times\left(\vec{r}-\vec{r}^{\prime}\right)=\rho d \phi\left[-z \cos \phi \hat{a}_{x}-z \sin \phi \hat{a}_{y}\right. \\
& \left.+(\rho-R \sin \phi) \hat{a}_{z}\right] . \\
& \mathrm{dH}_{z}=-\mathrm{j} \frac{\omega \mu \sigma \mathrm{N}_{T} \mathrm{~S}_{T} \mathrm{I}_{T}}{(4 \pi)^{2}} \\
& x \frac{\rho^{2}(\rho-R \sin \phi) d \phi d \rho d z}{\left(\rho^{2}+z^{2}\right)^{3 / 2}\left(\rho^{2}+z^{2}+R^{2}-2 R \rho \sin \phi\right)^{3 / 2}} .
\end{aligned}
$$

The response due to an eddy current loop can be found from

$\left(H_{z}\right)_{\text {Toop }}=\int_{\phi=0}^{\phi=2 \pi} d H_{z}$.

The response of a fracture existing from $z=z_{1}$ to $z=z_{2}$ can be found from

$\left(\mathrm{H}_{\mathrm{z}}\right)_{\text {fracture }}=\int_{\mathrm{Z}=\mathrm{Z}_{1}}^{\mathrm{Z}=\mathrm{Z}_{2}}\left(\mathrm{H}_{\mathrm{z}}\right)_{\text {loop }}$,

and the response of an infinite medium can be found by letting $z_{1}=\infty$ and $z_{2}=-\infty$ in Eq. (30). Equations (28), (29), and (30) can be applied to a vertically layered medium (i.e., $\sigma$ is a function of $z$ ) or a medium where $\sigma$ is a function of $\rho$ (horizontal cylinders), but not to cases where o varies with $\phi$. The latter case as well as on any other situation of boundary conditions that do not fit the coordinate system is not allowed because the symmetry was 
assumed in the formulation. For an infinite homogeneous medium, Eq. (30) combined with Eq. (21b) to yield the received signal due to eddy currents:

$V_{E}=-\left(\frac{\omega \mu}{4 \pi}\right)^{2} \sigma N_{T} S_{T} N_{R} S_{R} I_{T}$

$x \int_{Z=-\infty}^{\infty} \int_{\rho=0}^{\infty} \int_{\phi=0}^{2 \pi} \frac{\rho^{2}(\rho-R \sin \phi) d \phi d \rho d z}{\left(\rho^{2}+z^{2}\right)^{3 / 2}\left(\rho^{2}+z^{2}+R^{2}-2 R \rho \sin \phi\right)^{3 / 2}}$.

This compares with the eddy current signal of Eq. $(23 c)$ :

$V_{E}=-\frac{(\omega \mu)^{2}}{8 \pi R} \sigma N_{T} S_{T} N_{R} S_{R} I_{T}$

These two equations have the same functional dependence on $\mu, \omega$, and $\sigma$ (and with some algebra, R), as they should, but a direct analytic comparison depends on performing the integration of Eq. (31). This integration has not yet been completed analytically and is awaiting attack by a sufficiently clever mathematician. The integration has been done numerically, however, and this result has substantiated that Eqs. (31) and (32) are indeed the same. The details of this integration are not important here so they will be omitted. The importance of Eq. (31), however, is that it can be used to calculate the response of the two-coil instrument to an infinite fracture. This will be discussed in more detail in the next section.

B. Infinite Fracture Response

Equation (31) can be used directly to calculate the response of the two-coil instrument to an infinite fracture that is parallel to the plane of the coils and displaced horizontally from the coils a distance $z$. The received signal due to eddy currents in the fracture, $V_{E F}$, is:
$V_{E F}=-\left(\frac{\omega \mu \mu}{4 \cdot \pi}\right)^{2} \sigma t N_{T} S_{T} N_{R} S_{R} I_{T}$

$$
x \int_{\rho=0}^{\infty} \int_{\phi=0}^{2 \pi} \frac{\rho^{2}(\rho-R \sin \phi) d \phi d \rho}{\left(\rho^{2}+z^{2}\right)^{3 / 2}\left(\rho^{2}+z^{2}+R^{2}-2 R \rho \sin \phi\right)^{3 / 2}},
$$

where $t=z_{2}-z_{1}$ is the thickness of the fracture. Normalizing this equation by $R$ :

$V_{E F}=-\left(\frac{\omega \mu}{4 \pi R}\right)^{2}$ ot $N_{T} S_{T} N_{R} S_{R} I_{T}$

$x \int_{\frac{\rho}{R}=0}^{\infty} \int_{\phi=0}^{2 \pi} \frac{\left(\frac{\rho}{R}\right)^{2}\left[\frac{\rho}{R}-\sin \phi\right] d \phi d\left(\frac{\rho}{R}\right)}{\left[\left(\frac{\rho}{R}\right)^{2}+\left(\frac{Z}{R}\right)^{2}\right]^{3 / 2}\left[\left(\frac{\rho}{R}\right)^{2}+\left(\frac{Z}{R}\right)^{2}+1-2\left(\frac{\rho}{R}\right) \sin \phi\right]^{3 / 2}}$,

or substituting $x=\rho / R$ and $\gamma=z / R$ :

$$
\begin{aligned}
V_{E F}= & -\left(\frac{\omega \mu}{4 \pi T R}\right)^{2} \text { ot } N_{T} S_{T} N_{R} S_{R} I_{T} \\
& x \int_{x=0}^{\infty} \int_{\phi=0}^{2 \pi} \frac{x^{2}(x-\sin \phi) d \phi d x}{\left(x^{2}+\gamma^{2}\right)^{3 / 2}\left(x^{2}+\gamma^{2}+1-2 x \sin \phi\right)^{3 / 2}} .
\end{aligned}
$$

Let

$F(\gamma)=$

$$
\int_{x=0}^{\infty} \int_{\phi=0}^{2 \pi} \frac{x^{2}(x-\sin \phi) d \phi d x}{\left(x^{2}+\gamma^{2}\right)^{3 / 2}\left(x^{2}+\gamma^{2}+1-2 x \sin \phi\right)^{3 / 2}} .
$$


The function $F(\gamma)$ is shown in Fig. 7. Note that it is the function $F(\gamma)$ that must be integrated to yield the infinite medium results.

For a given fracture position, $Z$, the received signal will be proportional to the product of $\left(1 / R^{2}\right)$ and $F(Z / R)$. Thus, the instrument could be optimized for fractures of a given offset $Z$. Notice that the fracture position $Z=0$ is of most interest. At $Z=0$, the fracture is bisecting the borehole and it is under this condition that the instrument is desired to perform well. At $Z=0$, however, this analysis predicts that the received signal will be zero. The analys is is correct as confirmed by the comparison to the infinite medium response as was done earlier. This predicament must be overcome before an instrument of this type can be used to map fractures.

Heuristically, the zero response can be explained by viewing Fig. 8. The transmitting coil sets up an eddy current distribution independent of the position of the receiving coil according to the equations given here earlier. In relation to the receiver coil, these eddy currents comprise two classes: those that pass between the two coils (example of $I_{1}$ ) and those that do not (example of $I_{2}$ ). The magnetic fields of these two sets tend to cancel each other. Indeed, the integration performed to plot Fig. 7 indicates that they cancel exactly to zero. This observation provides a means of modifying the design so that the instrument will respond as desired. To make the instrument sensitive to fractures, where magnetic fields cancel as

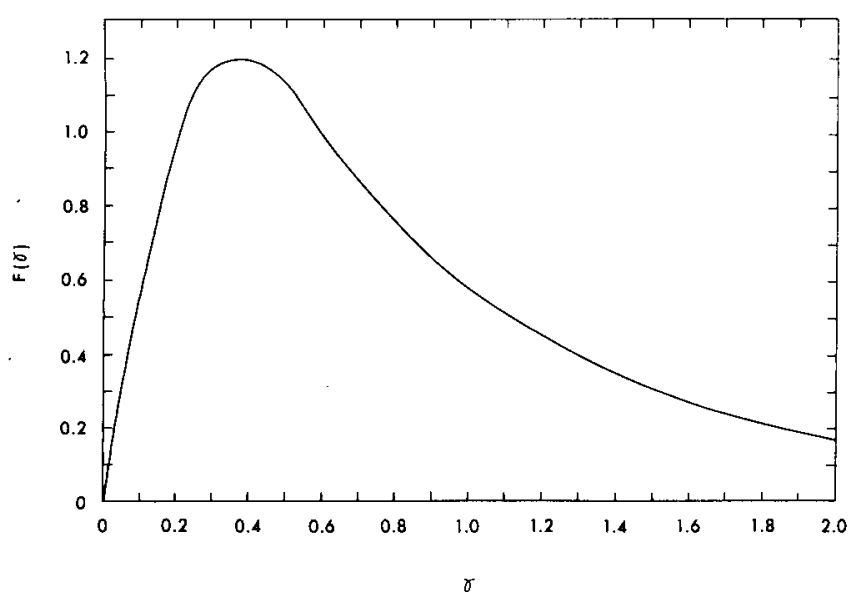

Fig. 7. The function $F(\gamma)$ for a thin infinite fracture.

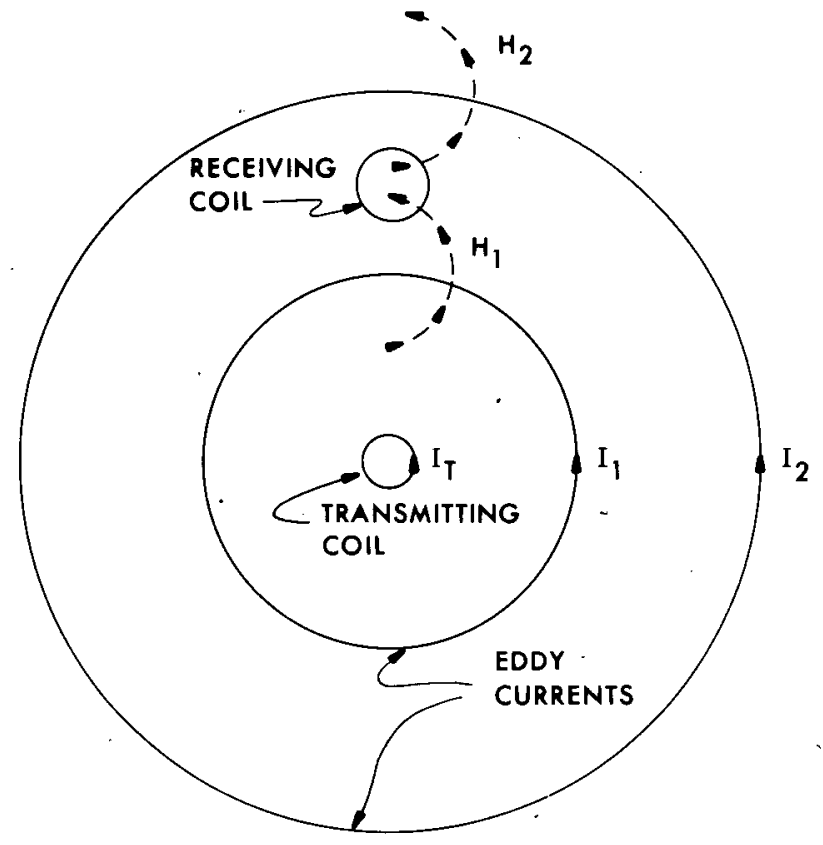

Fig. 8. Cancellation of the magnetic fields at the receiving coil.

in Fig. 8, a hole must be made in the fracture to stop the currents from flowing between the coils. Then, all the magnetic fields will add and the instrument will have a nonzero response at $Z=0$. Mechanically, this requires that the instrument be capable of displacing the conducting fluid in the borehole. This is also desirable because the borehole eddy current signal will be large if not interrupted.

C. Infinite Fracture with a Circular Hole

The response of a fracture with a circular hole centered around the transmitting coil can be found by using Eq. (33d) with the lower limit of integration set to the hole radius divided by $R$.

For a hole radius 10\% larger than the coil separation $R$, the response is shown in Fig. 9 as a function of fracture offset. Notice now that the maximum response is when the fracture is bisecting the borehole (i.e., at $Z=0$ ). The response can be increased by moving the coils together, but the depth of investigation increases as the coils are moved apart. 


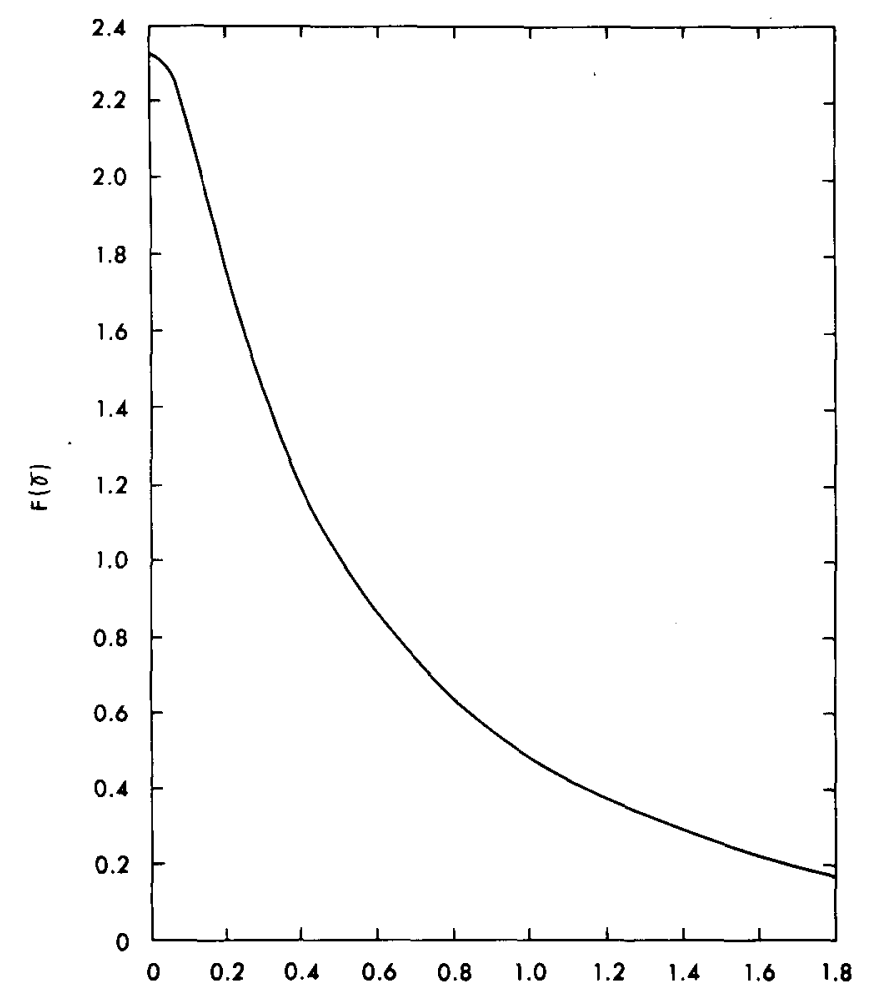

Fig. 9. The function $F(\gamma)$ for a fracture with a circular hole of radius 1.1R.

VI. THREE-COIL INSTRUMENT RESPONSE

A. Infinite Medium Response

A large "transformer" signal is measured with a two-coil instrument. This is an unwanted signal and is $90^{\circ}$ in phase from the desired signal. In theory, a phase-sensitive detector could discriminate against this unwanted signal. Because this transformer signal is much larger than the designed signal, the transformer signal is reduced by a technique commonly called "bucking."

In some instances, it is practical to affect the bucking electronically. For the present application, a more conventional approach will be used as shown with the three-coil system of Fig. 10 . The three coils are called the "transmitting coil," the "bucking coil," and the "receiving coil." If I is equal to $I_{B}$, then the received signal for a homogeneous medium (after Eq. 23c) is:
BOREHOLE AXIS

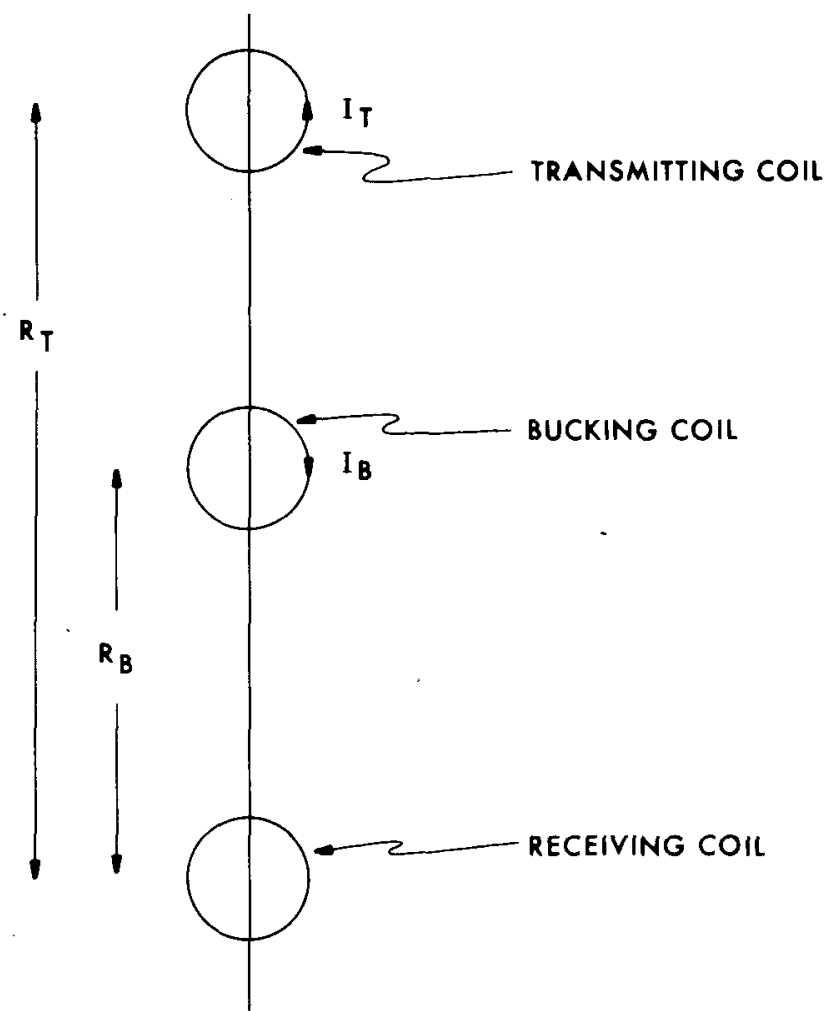

Fig. 10. Geometry of a three-coil instrument.

Because the transformer signal varies as $1 / R^{3}$ and the eddy current signal varies as $1 / R$, it is possible to cancel the transformer signal by choosing

$\frac{N_{T} S_{T}}{R_{T}{ }^{3}}=\frac{N_{B}{ }^{5} B}{R_{E}{ }^{3}}$

and not have the eddy current signal cancel as well. Several advantages are apparent for this "hardwired" bucking. If thermal expansion occurs, the bucking is not affected since it depends on ratios of distances. These ratios will not be affected if the thermal expansion is uniform. The placement of the bucking coil is not extremely critical. If there is an error in its placement such that

$V_{R}=j \frac{\omega \mu N_{R} S_{R} I_{T}}{4 \pi}\left[\left(\frac{N_{T} S_{T}}{R_{T}{ }^{3}}\right)-\left(\frac{N_{B} S_{B}}{R_{B}^{3}}\right)+j \frac{\omega \mu \sigma}{2}\left(\frac{N_{T} S_{T}}{R_{T}}-\frac{N_{B} S_{B}}{R_{B}}\right)\right]$. 
$R_{B}=(1+\alpha) R_{B 0}$,

(36a)

where $\alpha$ is the error about the location required for a null, it can be shown that the transformer signal is

$V=3 a V_{T}$,

where $V_{T}$ is the original transformer signal. A reduction in transformer signal of over 1000 times is relatively easy to achieve and maintain.

B. Three-Coil Response to a Fracture with a

\section{Rectangular Hole}

The three-coil instrument will still fail to respond to a fracture lying in the plane of the three coils. Consequently, a hole must be introduced in the fracture as was shown in Fig. 11. Shown here is the type of hole that can be generated in the fracture by filling the borehole with a nonconductive fluid retained by a rubber bladder. The analytic methods used here so far are not capable of solving for the instrument response of this

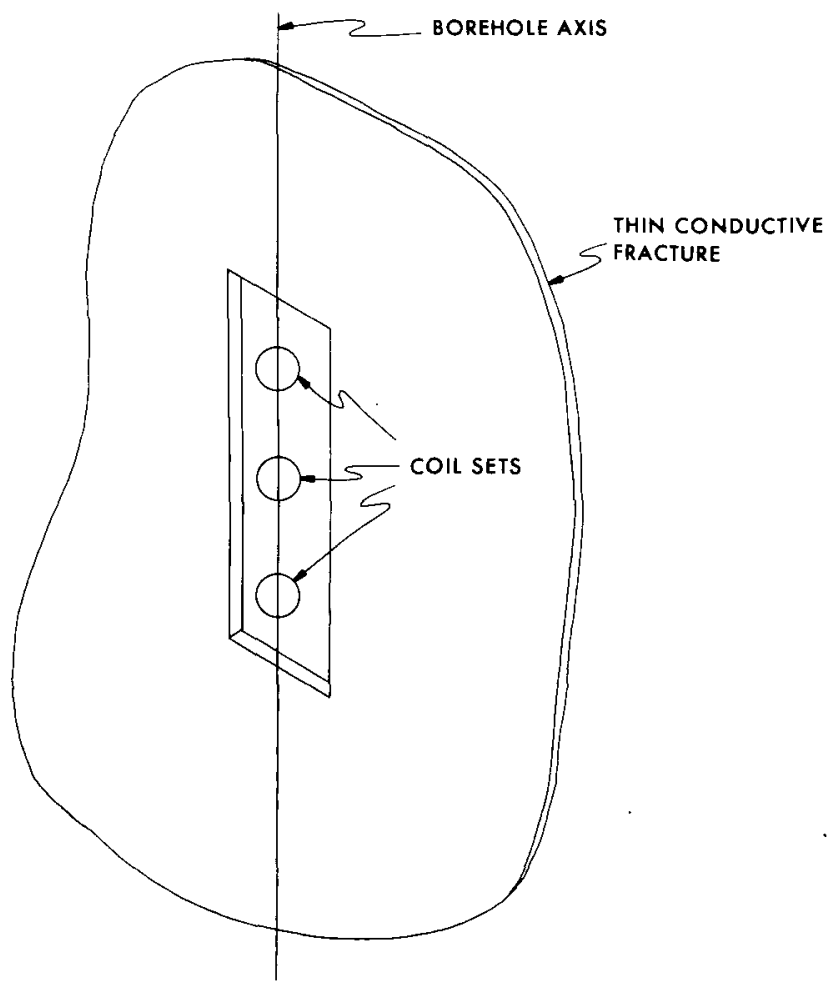

Fig. 11. Example of a rectangular hole created in a fracture by an oil-filled bladder.

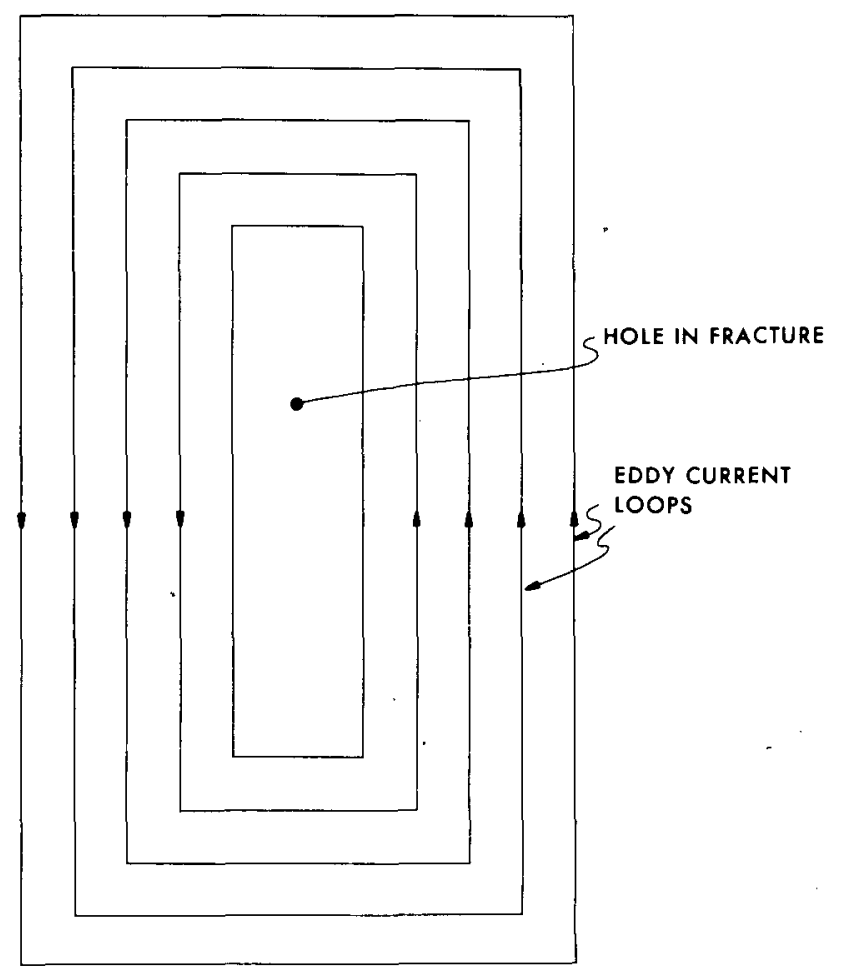

Fig. 12. Assumed eddy current distribution geometry around the rectangular hole in the fracture.

geometry. An approximation of the instrument response can be found, however, if an eddy current distribution is assumed. The accuracy of the dissolution willhinge on the accuracy of the assumed current distribution. Again, present programmatic requirements have precluded performing the complete analytic effort. However, an engineering quality approximation of instrument response $c a n$ be found using the current distribution shown in Fig. 12 . At large distances from the hole, this distribution reduces to a pattern of squares. The actual pattern at large distances is a series of circles. Near the hole, the assumed distribution matches the eddy current pattern fairly we 17 .

For the eddy current loop shown in Fig. 13a,

$\mathrm{I}_{\ell}=\frac{\mathrm{V}_{\ell}}{\mathrm{R}_{\ell}}$, 
(a)

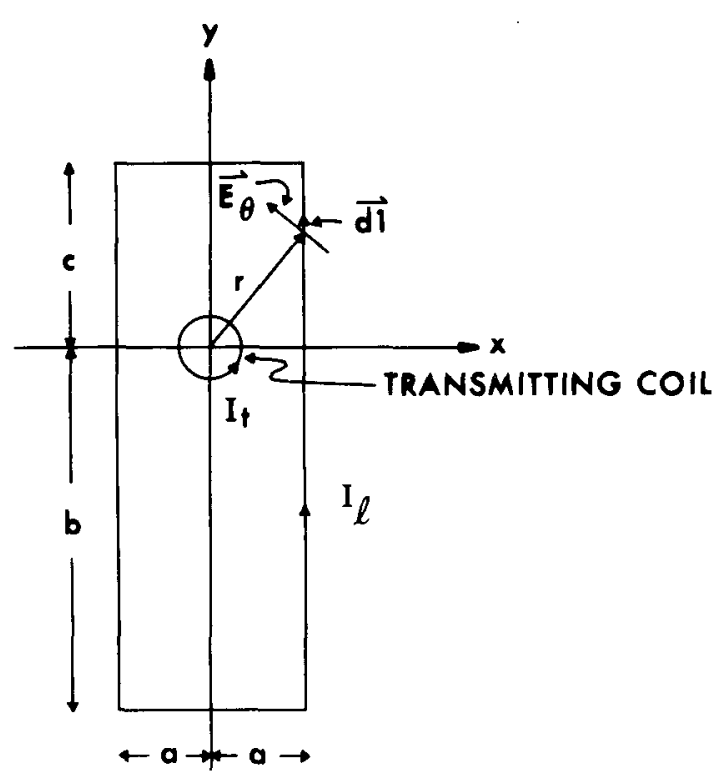

(b)

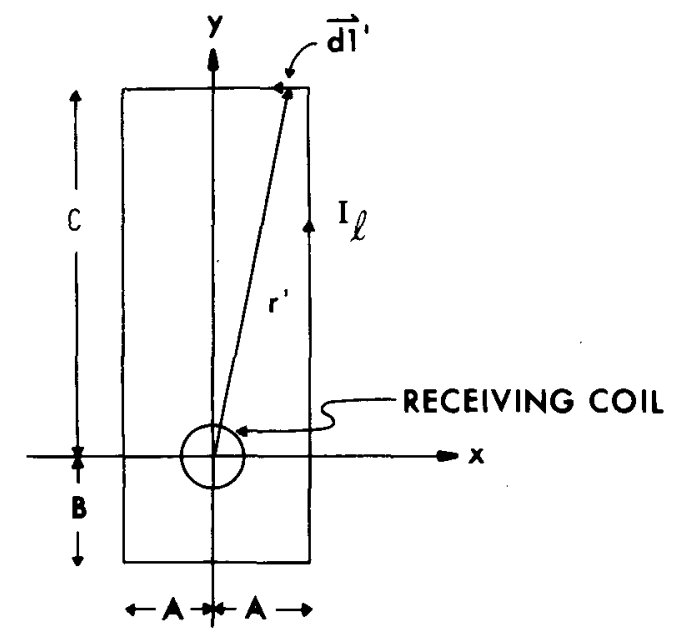

Fig. 13. Geometry for the rectangular eddy current distribution.

a. Transmitting

b. Receiving

$v_{\ell}=\oint \vec{E}_{\Theta} \cdot \overrightarrow{d l}$

$R_{\ell}=\frac{4(a+b)}{\sigma w t}$

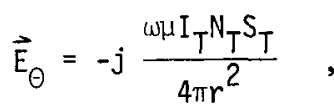

and where $t$ is the thickness and $w$ the width of the eddy current loop. Performing the integration of Eq. (37b),
$V_{\ell}=j \frac{\omega \mu N_{T} S_{T} I_{T}}{2 \pi}\left[-\sqrt{\frac{1}{a^{2}}+\frac{1}{c^{2}}}+\sqrt{\frac{l}{a^{2}}+\frac{1}{b^{2}}}\right]$,

where the parameters $a, b$, and $c$ are as shown in Fig. 13a. The receiving case geometry is shown in Fig. 13b. The magnetic field in the $Z$ direction can be found using Ampere's law (Eq. (5)), the geometry shown in Fig 13b, and the eddy current found in Eq. (37a):

$H_{Z}=\frac{I_{\ell}}{2 \pi}\left[\sqrt{\frac{1}{A^{2}}+\frac{1}{B^{2}}}+\sqrt{\frac{1}{A^{2}}+\frac{1}{C^{2}}}\right]$.

Then Eq. (21b) can be used to calculate the received signal. The response due to the fracture is then the summation of the signals due to al1 the eddy current loops. For the proposed three-coil system, the total voltage induced in each eddy current loop is the sum of that due to the transmitting coil and the bucking coil. The equations given here were checked with a simple experiment for a single eddy current loop made of a rectangular strand of wire with a series resistor. The measurements were found to agree with the prediction within $20 \%$. Also demonstrated experimentally was the $\cos ^{2} B$ dependence of signal, where $\beta$ is the angle between the plane of the eddy current loop (or fracture) and the plane of the coils.

VII. IDEAL INSTRUMENT RESPONSE

Previous sections have given various formulas, graphs, approximations, and discussions of the response of the proposed instrument. Here, these previous efforts will be used to predict the response given actual numbers for material properties and instrument design. The maximum diameter of the completed instrument is $0.15 \mathrm{~m}$ (6 in.), and enough $0 i 1$ must be carried in the instrument to inflate a bladder to reach a diameter of $0.25 \mathrm{~m}$ (10 in.). If oil extends one-half a coil spacing past each coil, and if the oil is contained in a reservoir of $0.15-m$ (6-in.) diameter, then the reservoir must be 5.6 times as long as the coil spacing. If the maximum coil spacing is $0.76 \mathrm{~m}(2.5 \mathrm{ft}$. or $30 \mathrm{in.})$, then the length of the reservoir is $4.3 \mathrm{~m}(14 \mathrm{ft})$. 
A]though a longer coil spacing is desirable for deep penetration into the rock, the need for carrying the oil along with the instrument limits the maximum coil spacing to about $0.76 \mathrm{~m}$. The eddy current signal depends on the frequency squared. An upper frequency of about $100 \mathrm{kHz}$ is set by the type of electronics needed for the phase-sensitive detector. At $100 \mathrm{kHz}$, the quasistatic condition is still valid and the fields are not markedly reduced by the "skin depth" effect.

To obtain quantitative results, the following design numbers will be used.

Coil spacing, transmitter to receiver, $R_{T}=$ $0.80 \mathrm{~m}$

Coil spacing bucking coil to receiver, $R_{B}=$ $0.40 \mathrm{~m}$

$\mathrm{N}_{T}=\mathrm{N}_{\mathrm{R}}=55$ turns

$S_{T}=S_{R}=2.3 \times 10^{-2} \mathrm{~m}^{2}$

$N_{B} S_{B^{\prime}}=\left(R_{B} / R_{T}\right)^{3} N_{T} S_{T}$ (for

perfect bucking)

Conductivity of rock, $\sigma_{R}=0.001 \mathrm{mho} / \mathrm{m}$

Conductivity of fracture, $\sigma_{\dot{F}}=0.1 \mathrm{mho} / \mathrm{m}$

Thickness of fracture, $t=1 \mathrm{~mm}$

Frequency, $\omega / 2 \pi=f=100 \mathrm{kHz}$

Dielectric constant of rock $=\varepsilon_{R}=9$,

where $\varepsilon=\varepsilon_{R} \varepsilon_{0}$

$\varepsilon_{0}=1 / 36 \pi \times 10^{-9}$

$\mu=\mu_{0}=4 \pi \times 10^{-7}$

transmit current $=I_{T}=I_{B}=10 \mathrm{~mA}$

The "background" signal from the granite can be found using Eq. (34).

$$
\begin{aligned}
V_{\text {rock }} & =-\frac{(\omega \mu)^{2} \sigma_{R} N_{R} S_{R} N_{T} S_{T} I_{T}}{8 \pi}\left(\frac{1}{R_{T}}-\frac{1}{8 R_{B}}\right) \\
& =-0.464 \mu \mathrm{V} .
\end{aligned}
$$

The "transformer" signal from the transmitting coil is

$$
\begin{aligned}
\left|V_{t}\right| & =\frac{\omega \mu N_{R} S_{R} N_{T} S_{T} I_{T}}{4 \pi R_{T}{ }^{3}} \\
& =1.134 \mathrm{mV} \\
& =2445 V_{\text {rock }} .
\end{aligned}
$$

Consequently, the bucking coil is required and maintaining a positioning accuracy of $1 / 2 \mathrm{~mm}$ will reduce the "transformer" signal to less than $2.6 \mu \mathrm{V}$, which is a reasonable level.

Estimation of the signal caused by fracture eddy currents may be accomplished using Eqs. (37a), $(37 e),(37 c),(38)$, and (21b). The response $V_{F}$ can be written as:

$V_{F}=-\left(\frac{\omega \mu}{2 \pi}\right)^{2}\left(N_{T} S_{T} N_{R} S_{R} \sigma t I_{T}\right) \int_{0}^{\infty} G(x) d x \cdot$

$G(x)$ is commonly referred to as the "geometrical factor" and depends, in this case, on the coil locations and the size of the hole in the fracture. Here, $x$ refers to distance away from the borehole wa11. It is instructive to look at $G(x)$ and $\int_{0}^{x} G(x) d x$. For the three-coil set of the previous examples with the bucking coil centered in the rectangular hole in the fracture, $G(x)$ is shown in Fig. 14a and $\int_{0}^{X} G(x) d x$ in Fig. 14b. A rectangular hole $0.25 \mathrm{~mm}$ (9.8 in.) wide and $1.4 \mathrm{~m}$ (55 in.) tall was used in Fig. 14. $G(x)$ was obtained straightforwardly from the equations listed above and $\int_{0}{ }^{X} G(x) d x$ was obtained by numerical integration.

Figure 14 shows the major response comes from quite near the borehole; $50 \%$ of the response originates within $0.09 \mathrm{~m}(4 \mathrm{in.})$ of the borehole wall
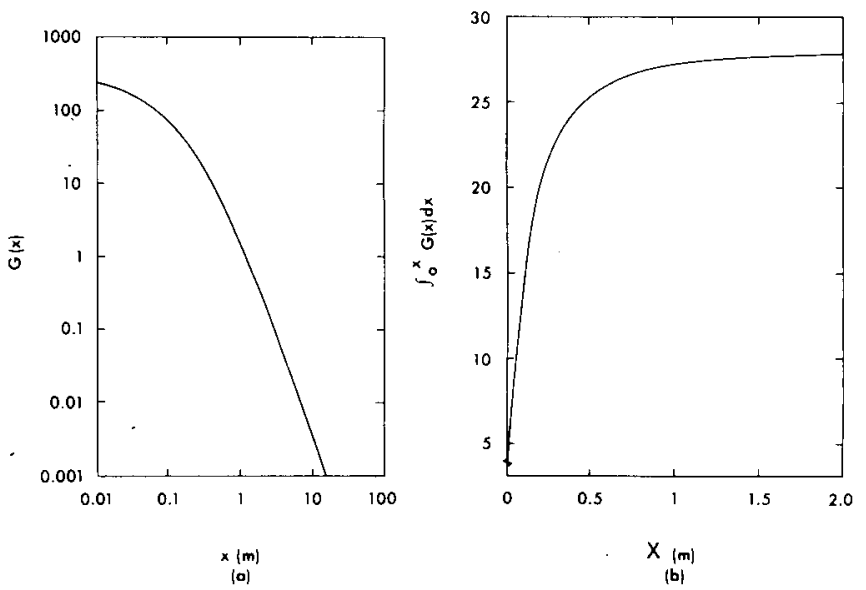

Fig. 14. Response of a three-coil instrument to a fracture with a rectangular hole.

a. The geometrical factor.

b. The integral of the geometrical factor. 
and $90 \%$ from within $0.45 \mathrm{~m}$ (18 in.). Deeper investigation can be obtained with larger coil separations or a multicoil system to "focus" the response similarly to current practice in induction $\log$ devices used by the oil industry. For the case under present consideration, the fracture response is

$V_{F}=0.177 \mu V$

The response will be increased if the fracture is wider than $1 \mathrm{~mm}$ and the conductivity is greater than $0.1 \mathrm{mho} / \mathrm{m}$. If both of these parameters are too small by a factor of 3 , the fracture signal is $V_{F}$ $=1.053 \mathrm{~V}$. In any case, the electronics circuitry being constructed (and to be reported separately) is capable of measuring these expected signals.

\section{OTHER DESIGN CONSIDERATIONS}

Many factors have been ignored in the previous analysis. Some of these will be discussed briefly here.

of largest concern is the borehole signal. The signal varies approximately as the volume of the conductive material supporting the eddy currents and roughly as the inverse cube of distance from the coils. With these rough guidelines, the borehole signal will be at least 10 times as large as the desired signal. If the instrument is not centered in the borehole, or if the borehole is noncircular, the resulting asymmetries will swamp out the desired signal originating in the fracture. Consequently, it is imperative that the borehole signal is eliminated by displacing the conductive borehole fluid as discussed earlier. The guidelines given here also provide estimates of how well the borehole fluid must be displaced. Small pockets of fluid will not be detected since the eddy current paths are too small. Larger voids should contain less conductive material than is in the fracture within roughly $1 / 2 \mathrm{~m}$ from the borehole. This means that the conductive fluid should be displaced, leaving an annulus with no more than about a 1-mm thickness of fluid.

A second class of problems yet to be addressed are planar fractures with orientations and locations different than considered here in previous sections.
The effects of fracture tilt and offset should be studied. The response of nonplanar fractures is also of interest.

A third class of problems deals with the construction of the instrument. Extreme care must be exercised in grounding and shielding. The effective shielding and isolation between the transmitter and receiver must be at least $160 \mathrm{~dB}$ !! This is obtainable with careful shielding and grounding as has been demonstrated by laboratory working models.

\section{SUMMARY}

The proposed three-coil instrument will map the type of fractures anticipated as long as the conductive'borehole fluid can be displaced. This report has been a somewhat rambling analys is of the instrument. The analysis has been fairly complete but at a fairly elementary level. More analyses should be undertaken, but the next level of analys is involves much more effort.

Additional studies can be performed using the concepts presented here. Instruments containing more than three coils could be considered. An impicit assumption in this entire report is that the coils used in the instrument are "small." The actual coils are not small, however, with respect to the dimensions encountered. Experimentation has indicated that no large errors can be attributed to the use of the "small" coil equations, but this should be considered more fully.

Finally, more model testing and experimentation should be performed. The experiments conducted to date have been fairly simple and under laboratory conditions. It is essential that tests be performed in rock and with real fractures to gain confidence in this analysis. This should be done before the instrument is lowered $3000 \mathrm{~m}$ into the ground.

\section{ACKNOWLEDGMENTS}

The fracture mapping method described here was conceived at the LoS Alamos Scientific Laboratory by $P$. Kintzinger, F. West, and W. Johnson. The continued development has been made possible through the support of A. Blair and J. Rowley. Funding was provided by ERDA through the Hot Dry Rock Geothermal Energy Development Project. 


\section{REFERENCES}

1. "The LoS Alamos Scientific Laboratory's Dry Hot Rock Experiment: Engineering and Scientific Studies," a series of papers by LASL HDR staff, presented at the 1976 Spring Meeting of the American Geophysical Union, Washington, DC, April 12-15, 1976, Los Alamos Scientific Laboratory unpublished material, Los Alamos, NM.

2. J. H. Moran and K. S. Kunz, "Basic Theory of Induction Logging and Application to Study of Two-Coil Sondes," Geophysics, Vol. 27, No. 6, pp. 829-858, December 1962.

3. H B. Watt, G. W. Hammack, H. Guyod, P. A. Wichmann, L. E. Schneider, R. D. Wood, 0 . R. Holt, D. W. Hilchie, Log Review 1, Dresser Industries, Inc., Dallas TX (1974).

4. For a description of terms, see: R. C. Ransom, "Glossary of Terms and Expressions Used in Well Logging," Society of Professional Well Log Analysts, Houston, TX, 1975.

5. P. R. Kintzinger, "Seismic Signal Location Program for the Los Alamos Scientific Laboratory's Dry Hot Rock Geothermal Project," LA-6274-MS, Los Alamos Scientific Laboratory, March 1976.
6. B. Dennis, J. Hill, E. Stephani, and B. Todd, "Development of High-Temperature Acoustic Instrumentation for Characterization of Hydraulic Fractures in Dry Hot Rock," Los ATamos Scientific Laboratory internal document (May 1976).

7. D. V. Power, C. L. Schuster, R. Hay, and J. Twombly, "Detection of Hydraulic Fracture Orientation and Dimensions in Cased Wells," Journal of Petroleum Technology, pp. 1116-1124, September 1976.

8. J. D. Byerlee and M. J. S. Johnston, "A Magnet ic Method for Determining the Geometry of Hydraulic Fractures," Pageoph, Vol. 114, pp. $425-433,1976$.

9. R. F. Harrington, Time-Harmonic Electromagnet ic Fields, (McGraw-Hil1 Book Co., New York, NY, 1961).

10. J. R. Wait, "Electromagnetic Fields of Sources in Lossy Media," in Antenna Theory, R. E. Collin and F. J. Zucker, Eds. (MCGraw-Hill Book Co., New York, NY, 1969).

11. J. A. Fuller and J. R. Wait, "EM Coupling of Coaxial and Coplanar Loops in Uniform Dissipative Media," IEEE Proc., pp. 993-994, (August 1972). 
Printed in the United States of America. Availuble from National Technical Information Service

lis Department of Commerce

5285 Port Royal Road

Springtield, VA 22161

Mictotiche $\$ 3.00$

$\begin{array}{llllllllll}001-025 & 4.00 & 126-150 & 7.25 & 251-275 & 10.75 & 376-400 & 13.00 & 501-525 & 15.25 \\ 026-050 & 4.50 & 151-175 & 8.00 & 276-300 & 11.00 & 401-425 & 13.25 & 526-550 & 15.50 \\ 051-075 & 5.25 & 176-200 & 9.00 & 301-325 & 11.75 & 426-450 & 14.00 & 551-575 & 16.25 \\ 076-100 & 6.00 & 201-225 & 9.25 & 326-350 & 12.00 & 451-475 & 14.50 & 576-600 & 16.50 \\ 101-125 & 6.50 & 226-250 & 9.50 & 351-375 & 12.50 & 476-500 & 15.00 & 601-110 & \end{array}$

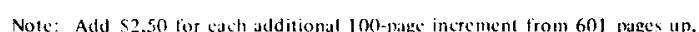

